\title{
Magnetic ordering in Fe/Co sandwiches on $\mathrm{Cu}(\mathbf{1 0 0})$
}

\author{
S S A Razee ${ }^{1,4}$, J B Staunton ${ }^{2}$ and L Szunyogh ${ }^{3}$ \\ ${ }^{1}$ Department of Physics, Kuwait University, PO Box 5969, Safat 13060, Kuwait \\ ${ }^{2}$ Department of Physics, University of Warwick, Coventry CV4 7AL, UK \\ ${ }^{3}$ Department of Theoretical Physics, Budapest University of Technology and Economics, \\ H-1521 Budapest, Hungary \\ E-mail: razee@physics.kuniv.edu.kw
}

Received 23 February 2009, in final form 13 May 2009

Published 11 June 2009

Online at stacks.iop.org/JPhysCM/21/265004

\begin{abstract}
We investigate magnetic correlations and local magnetic moments at finite temperatures of some $\mathrm{Fe}$ and $\mathrm{Co}$ multilayers on $\mathrm{Cu}(100)$ substrates, such as $\mathrm{Co}_{m} \mathrm{Fe}_{n} \mathrm{Co}_{m} / \mathrm{Cu}(100)$ and $\mathrm{Fe}_{m} \mathrm{Co}_{n} \mathrm{Fe}_{m} / \mathrm{Cu}(100)$. We use an ab initio mean-field theory of magnetic fluctuations for layered materials based on the first-principles local spin-density functional theory implemented through the screened Korringa-Kohn-Rostoker method. We find that the presence of Fe layers in the neighbourhood of a Co layer always leads to a reduction in the magnetic moment of the Co atoms, whereas that of the $\mathrm{Fe}$ atoms is enhanced. Of particular interest is the lack of local moment formation on the single fcc-Co layer sandwiched between two fcc-Fe layers. However, a Co layer completely immersed in a $\mathrm{Cu}$ environment remains ferromagnetic. The Curie temperature of the $\mathrm{Co}_{m} \mathrm{Fe}_{n} \mathrm{Co}_{m} / \mathrm{Cu}(100)$ system oscillates as the Fe layer thickness is increased whereas that of the $\mathrm{Fe}_{m} \mathrm{Co}_{n} \mathrm{Fe}_{m} / \mathrm{Cu}(100)$ system increases almost monotonically with Co layer thickness.
\end{abstract}

\section{Introduction}

Magnetic nanostructures in the form of thin films and multilayers containing ferromagnetic transition metals have been a subject of intense research due to their immense potential as materials for 'next-generation' high-tech applications [1-4]. In particular, creating ultra-high density media for future magnetic storage applications is one of the biggest challenges in the field of nanomagnetism. In order to advance to a storage density of Tbits/inch ${ }^{2}$ and beyond it is proposed to use heat-assisted magnetic recording or thermomagnetic recording. But thermal stability of small structures [5] and design and sensitiveness of read-head sensors based on giant magnetoresistive effects [6] are major obstacles. Therefore, it is important to study not only the ground state magnetic properties, but the magnetic properties at higher temperatures as well of these thin magnetic multilayers. From a purely fundamental physics point of view also these 'thin magnets' are of great interest because of their exotic properties. A great deal of experimental data for these materials is available in the literature [7]: however, theoretical calculations, especially from a first-principles elec-

4 Author to whom any correspondence should be addressed. tronic structure point of view, are few because of the complex nature of the problem. Theoretically, the 'disordered local moment' (DLM) picture [8-11] is probably the only theory for the study of the onset of magnetic order at finite temperatures which has a strong appeal as well as being suitable to be implemented within first-principles electronic structure models. It is based on the premise that there is a separation between fast and slow electronic degrees of freedom. For times long in comparison with electronic 'hopping' times, but short when compared with typical spin fluctuation times, the spin orientations of the electrons leaving an atomic site are sufficiently correlated with those arriving at that site so that the magnetization integrated over a unit cell and averaged over time is non-zero. These are the 'local moments' which change their orientations on a longer timescale while their magnitudes fluctuate rapidly on this timescale.

This basic picture has been applied before to bulk materials [12, 13] and, most recently, to thin films and multilayers [14-17] within first-principles electronic structure models without an intermediate step of mapping to an effective Heisenberg model. In our study of some magnetic thin films $[14,15,17]$ we found that the layer dependence of the electronic structure of the magnetic films on or embedded 
in a non-magnetic substrate drives the onset of magnetic order and the form of magnetic interactions between the magnetic layers. It was found that, in the $\mathrm{Cu}_{m} \mathrm{Fe}_{n} / \mathrm{Cu}(100)$ films, the $T_{\mathrm{c}}$ oscillates as a function of the $\mathrm{Cu}$ capping-layer thickness $(m)$ [14] with a maximum for the uncapped film in agreement with experiment [18]. This behaviour is attributed to the fact that in the uncapped film the top two Fe layers are coupled ferromagnetically while the nearest-neighbour interlayer coupling within the film is antiferromagnetic. In the presence of a capping layer all the interlayer couplings become antiferromagnetic [14]. However, the intralayer couplings always remain strongly ferromagnetic. In the case of $\mathrm{Cu}_{m} \mathrm{Co}_{n} / \mathrm{Cu}(100)$ films the capping layers had a steadying effect on $T_{\mathrm{c}}$ although the first capping layer did produce a sharp decrease in the $T_{\mathrm{c}}$ [15]. It was also observed [17] that the effect of capping by a ferromagnetic material, such as Co, on the $\mathrm{Fe}_{n} / \mathrm{Cu}(100)$ films was only to monotonically increase the $T_{\mathrm{c}}$ as the capping-layer thickness was increased. This is probably due to the fact that the nature of interlayer as well as intralayer magnetic correlations remained unchanged although the ferromagnetic couplings became stronger.

The DLM theory has also been extended to study the temperature dependence of magnetic anisotropy. As the material is annealed from its paramagnetic state from a temperature high above its $T_{\mathrm{c}}$, the local moments begin to align themselves along a favourable direction. So the magnetic anisotropy starts to grow as the temperature gradually approaches $T_{\mathrm{c}}$ from above. Since magnetic anisotropy is a relativistic phenomenon, the DLM approach has been generalized recently to take the relativistic effects into account $[19,20]$ and was used successfully to study the temperature dependence of the magnetic anisotropy constant of FePt. This relativistic DLM scheme has also been extended to calculate the temperature dependence of the magnetic anisotropy energy of thin films [21, 22]. Strong correlation effects have also been incorporated as shown by studies of the heavy rare earths [23] and some transition metal oxides [24].

In the present work, we have used the spin-polarized screened Korringa-Kohn-Rostoker (SKKR) coherent-potential approximation (CPA) method [25-27] for layered systems adapted for the DLM approach to study the magnetic properties of some $\mathrm{Co}_{m} \mathrm{Fe}_{n} \mathrm{Co}_{m} / \mathrm{Cu}(100)$ and $\mathrm{Fe}_{m} \mathrm{Co}_{n} \mathrm{Fe}_{m} / \mathrm{Cu}(100)$ multilayered films. Our interest in these films stems from a recent proposal [28] to use FeCo alloy films as the material for high density magnetic recording devices rather than the FePt alloys, the former favourites. This is because, at specific structural distortions, FeCo alloys have been predicted to have a large saturation magnetization as well as a large uniaxial magnetic anisotropy. But for heat-assisted magnetic recording, which is the projected technique for next-generation magnetic recording, the Curie temperature is also an important factor. We find that these films as a whole are ferromagnetic although some interlayer magnetic correlations, especially between two Fe layers on either side of a Co layer, may be antiferromagnetic in nature. We start with the paramagnetic state in which the local moments have disordered orientations, making the system paramagnetic overall. The local magnetic moment of a Co layer decreases substantially if it is close to a Fe layer whereas that of the corresponding Fe layer increases. In particular, there is no local moment formation on a single Co layer sandwiched between two Fe layers. This is interesting because a Co layer in a completely non-magnetic environment such as the one deep inside $\mathrm{Cu}$ still remains ferromagnetic. Previously, some magnetically dead layers were observed when a single layer of a ferromagnetic transition metal was immersed in a non-magnetic environment, such as $\mathrm{Co}$ in $\mathrm{Nb}$ and $\mathrm{Fe}$ in $\mathrm{Nb}$ [29-31], Ni in Pt [32], etc. In the present case, although there is no local moment formation in the Co layer, it will be premature to call this Co layer magnetically dead because of the following reasons. Firstly, two Co layers are sandwiched between Fe layers exhibiting formation of local moments. So it might be the case that a $\mathrm{Co}$ atom requires a sufficient number of Co atoms around it to form local moments. Secondly, $T=0$ calculations for the $\mathrm{Fe}_{1} \mathrm{Co}_{1} \mathrm{Fe}_{1} / \mathrm{Cu}(100)$ film show that, when the two Fe layers are ferromagnetically coupled, there is some magnetic moment on the Co layer parallel to those of the Fe layers. The situation of the two Fe layers coupled antiferromagnetically is unstable at $T=0$. Our DLM calculations indicate that the two Fe layers on either side of the Co layer might be antiferromagnetically coupled. This makes the present case very interesting and needs experimental investigation. The Curie temperature of these films also exhibit some oscillatory behaviour as the thickness of the sandwiched layer is increased.

This paper is organized as follows. In section 2 we briefly outline the theoretical framework and give some details of the computational methods used. In section 3 we present our results and in section 4 we present the conclusions.

\section{Theoretical framework and computational details}

Our study of finite temperature magnetism of thin films and multilayers is based on the DLM picture, in which the local moments are set up as a consequence of a separation between the collective behaviour of the interacting itinerant electrons and a much slower variation in the orientations of the spin of these electrons. Below the transition temperature, the orientations are correlated enough to give rise to an ordered magnetic structure. For theoretical and computational details the reader is referred to [15]. In the following, we give a brief outline of some of the features of our calculations.

The key quantity to these calculations is the layeredresolved paramagnetic spin susceptibility at temperature $T$, given by

$$
\mathcal{X}_{\mathrm{PQ}}\left(\mathbf{q}_{\|}\right)=\mu_{\mathrm{P}}^{2}\left[\left(3 k_{\mathrm{B}} T\right) I-\mathcal{S}^{(2)}\left(\mathbf{q}_{\|}\right)\right]_{\mathrm{PQ}}^{-1}
$$

where $\mathbf{q}_{\|}$is a wavevector in a layer, $\mu_{\mathrm{P}}$ is the magnitude of the local magnetic moment in layer $\mathrm{P}$ and $k_{\mathrm{B}}$ is the Boltzmann constant. The matrix elements $\mathcal{S}_{\mathrm{PQ}}^{(2)}\left(\mathbf{q}_{\|}\right)$are the twodimensional lattice Fourier transforms of the "direct correlation functions' given by

$$
\mathcal{S}_{i j}^{(2)}=-\left.\frac{\partial^{2} \Omega}{\partial m_{i} \partial m_{j}}\right|_{\left\{m_{i}=\bar{m}_{i}\right\}}
$$

where $\Omega$ is the mean-field averaged generalized electronic grand potential consistent with the spin-density functional 
theory, $m_{i}$ is the magnetization on site $i$ and $\bar{m}_{i}$ is the average of $m_{i}$ over the orientations of the local moments. Within a nonrelativistic electronic structure framework, the DLM model maps readily onto a random binary alloy $\mathrm{A}_{0.5} \mathrm{~B}_{0.5}$ with the species A and B representing the 'up-spin' and 'down-spin' sites, respectively. Therefore thermal spin fluctuations can be handled using the 'CPA' for layered systems. With this approximation, $\mathcal{S}_{\mathrm{PQ}}^{(2)}\left(\mathbf{q}_{\|}\right)$can be expressed as a convolution integral in the surface Brillouin zone. However, for the study of ferromagnetic transitions, we need to calculate $\mathcal{S}_{\mathrm{PQ}}^{(2)}\left(\mathbf{q}_{\|}\right)$for $\mathbf{q}_{\|}=0$ only. As outlined in [17] $\mathcal{S}_{\mathrm{PQ}}^{(2)}\left(\mathbf{q}_{\|}=0\right)$ is related to the variation of 'Weiss field' $\mathcal{S}_{\mathrm{P}}^{(1)}$ in layer $\mathrm{P}$ with respect to the change in the magnetization on layer $Q$ :

$$
\mathcal{S}_{\mathrm{PQ}}^{(2)}\left(\mathbf{q}_{\|}=0\right)=-\frac{\partial \mathcal{S}_{\mathrm{P}}^{(1)}}{\partial m_{\mathrm{Q}}} .
$$

We have used a multiple scattering approach, the SKKR method, to calculate the electronic structure from first principles. In the SKKR-CPA formalism

$$
\mathcal{S}_{\mathrm{P}}^{(1)}=-\frac{1}{\pi} \operatorname{Im} \int_{-\infty}^{\infty} \mathrm{d} \varepsilon f(\varepsilon, v)\left[\ln \left\|D_{\mathrm{P}}^{\uparrow}\right\|-\ln \left\|D_{\mathrm{P}}^{\downarrow}\right\|\right]
$$

where $f(\varepsilon, v)$ is the Fermi factor with chemical potential $v$ and

$$
D_{\mathrm{P}}^{\uparrow(\downarrow)}=\left[I+\left\{\left(t_{\mathrm{P}}^{\uparrow(\downarrow)}\right)^{-1}-\left(t_{\mathrm{P}}\right)^{-1}\right\} \tau_{\mathrm{P}}^{00}\right]^{-1}
$$

satisfy the CPA condition:

$$
D_{\mathrm{P}}^{\uparrow}+D_{\mathrm{P}}^{\downarrow}=2 I .
$$

In the above equations, $\tau_{\mathrm{P}}^{00}$ are the site-diagonal CPA 'pathoperator' matrices, $t_{\mathrm{P}}^{\uparrow(\downarrow)}$ is the scattering matrix of the up (down) species and $t_{\mathrm{P}}$ is the scattering matrix of the CPA effective medium, all for layer P. Equation (6) needs to be solved self-consistently.

When the system is cooled down from a high temperature, the ferromagnetic order starts around the temperature at which the instabilities in the spin fluctuations diverge. So at the Curie temperature

$$
\left\|\left(3 k_{\mathrm{B}} T_{\mathrm{c}}\right) I-\mathcal{S}^{(2)}\left(\mathbf{q}_{\|}=0\right)\right\|=0 .
$$

This implies that the largest positive eigenvalue of $\mathcal{S}^{(2)}\left(\mathbf{q}_{\|}=\right.$ $0)$ is related to $T_{\mathrm{c}}$ :

$$
T_{\mathrm{c}}=\frac{\text { Largest positive eigenvalue of } \mathcal{S}^{(2)}\left(\mathbf{q}_{\|}=0\right)}{3 k_{\mathrm{B}}} .
$$

We point out that the first-principles DLM theory outlined above is a mean-field theory, hence it is expected to overestimate the $T_{\mathrm{c}}$ for layered systems. In particular, in the ultrathin (two-dimensional) limit $(n=1,2)$ the meanfield treatment of fluctuations is expected to have its greatest shortcomings. For example, it does not reproduce the empirical scaling behaviour [33], $T_{\mathrm{c}}(n) \propto n^{-\lambda}$, with $\lambda$ being a nonuniversal scaling exponent. Nevertheless, the trends in the $T_{\mathrm{c}} \mathrm{s}$ with respect to the layer composition, such as the thickness of the magnetic film $(n \geqslant 3)$ and the thickness of the nonmagnetic capping film, are expected to be well described as evidenced from previous works [14-17].

In our calculations, the effective scattering potentials and the exchange fields of the multilayers were determined self-consistently by using the SKKR-CPA method [25-27]. In all cases the exchange-correlation energy of the KohnSham spin-density functional theory was calculated using the Perdew and Zunger parametrization of the local spin-density approximation of Ceperly and Alder [34]. All the films are treated as perfect face-centred cubic structures deposited layer by layer on the substrate without interdiffusion. We have used the lattice parameters of the substrate, fcc-Cu (6.83 au), and the effects of lattice strain on the electronic structure are ignored. For each film, the electronic structure of each layer of the film, as well as that of a buffer of three layers of the substrate and a buffer of at least three layers of vacuum, was calculated selfconsistently. The local spin densities were calculated within the atomic-sphere approximation in which all the atoms were assumed to have the same atomic radii as that of the $\mathrm{Cu}$ atoms. In the calculation of the spin densities and local moments, the required energy integration was performed by using 25 complex energy points along a semicircular contour spanning the bottom of the band and the Fermi energy. The Brillouin zone integration was performed by using $45 k_{\|}$points in the irreducible part of the surface Brillouin zone. This ensures an accuracy of about $10^{-3} \mu_{\mathrm{B}}$ for the calculated local moments.

\section{Results and discussion}

We have investigated a few $\mathrm{Co}_{m} \mathrm{Fe}_{n} \mathrm{Co}_{m} / \mathrm{Cu}(100)$ and $\mathrm{Fe}_{m} \mathrm{Co}_{n} \mathrm{Fe}_{m} / \mathrm{Cu}(100)$ systems for $m$ and $n=0$, 2 and 3. In section 3.1 we present the results for $\mathrm{Fe}_{m} \mathrm{Co}_{n} \mathrm{Fe}_{m} / \mathrm{Cu}$ (100) films and in section 3.2 we present the results for $\mathrm{Co}_{m} \mathrm{Fe}_{n} \mathrm{Co}_{m} / \mathrm{Cu}(100)$ films.

\section{1. $\mathrm{Fe}_{m} \mathrm{Co}_{n} \mathrm{Fe}_{m} / \mathrm{Cu}(100)$ films}

These multilayers are of fundamental importance because of their exotic properties. These provide a stringent test of theoretical models because of their 'clean' interface structure due to the fact that $\mathrm{Fe}$ and $\mathrm{Cu}$ are immiscible, and so there is almost no interdiffusion with the substrate, resulting in pure films. In a previous work, we studied $\mathrm{Co}_{n} \mathrm{Fe}_{m} / \mathrm{Cu}$ (100) films in which we found that the thickness of the Co layer on Fe films helps in stabilizing the ferromagnetic behaviour of the films, resulting in an increase of the local magnetic moments as well as the Curie temperature of the films. In this work we have studied the sandwich structure $\mathrm{Fe}_{m} \mathrm{Co}_{n} \mathrm{Fe}_{m} / \mathrm{Cu}(100)$ with $n$ and $m$ varying between 0 and 3 . The local magnetic moments and the Curie temperatures of these films are presented in table 1. The interesting feature of these films is that the Co layer close to the $\mathrm{Fe}$ layer loses some of its magnetic moment while the corresponding Fe layer gains. In particular, in the $\mathrm{Fe}_{m} \mathrm{Co}_{1} \mathrm{Fe}_{m} / \mathrm{Cu}(100)$ films the magnetic moment of the Co layer is almost zero for all Fe layer thicknesses. In other words, local moments do not form on a single Co layer sandwiched between two Fe layers while the film is still ferromagnetic as 

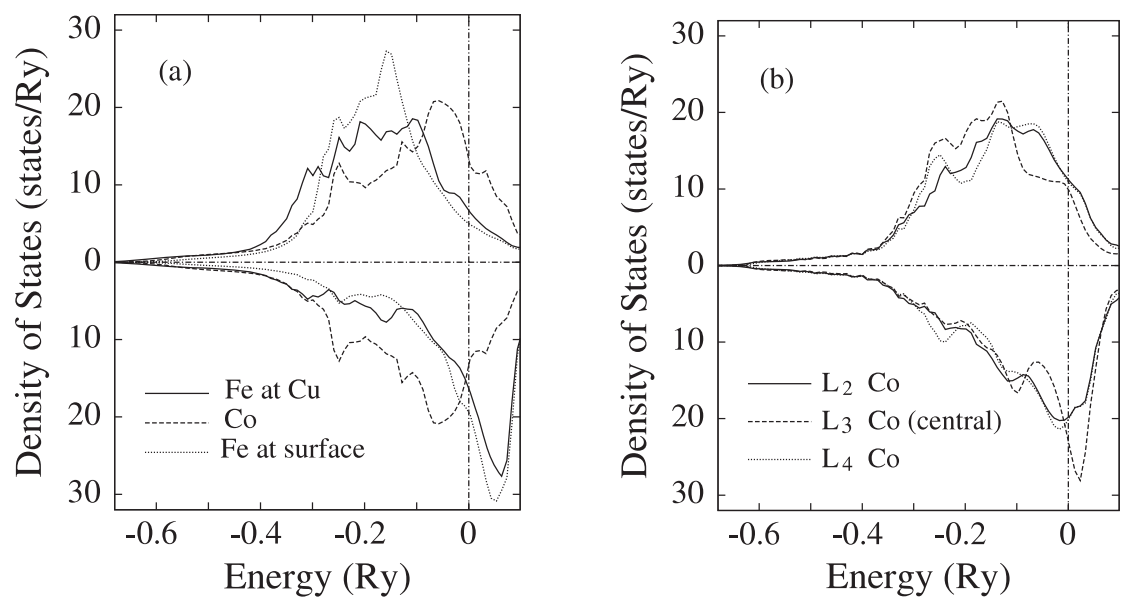

Figure 1. (a) Electronic densities of states for some selected layers of the $\mathrm{Fe}_{1} \mathrm{Co}_{1} \mathrm{Fe}_{1} / \mathrm{Cu}(100)$ film in the DLM state. The full line represents the Fe layer at the substrate, the dotted line represents the Fe layer at the surface and the dashed line represents the Co layer. (b) Electronic densities of states in the three Co layers of the $\mathrm{Fe}_{1} \mathrm{Co}_{3} \mathrm{Fe}_{1} / \mathrm{Cu}(100)$ film in the DLM state. The full line represents the Co layer closest to the substrate, the dashed line represents the central Co layer, while the dotted line represents the Co layer closest to the vacuum. In both figures, the upper (lower) half shows the density of states for an electron spin-polarized parallel (anti-parallel) to the local moment on a site. The energy is measured from the Fermi energy.

Table 1. The local magnetic moments on different layers and the Curie temperatures of $\mathrm{Fe}_{m} \mathrm{Co}_{n} \mathrm{Fe}_{m} / \mathrm{Cu}(100)$ films. Note that the layer $\mathrm{L}_{1}$ (the rightmost in the film title) is adjacent to the substrate and the leftmost layer is at the surface.

\begin{tabular}{|c|c|c|c|c|c|c|c|c|c|c|}
\hline \multirow[b]{2}{*}{ Films } & \multicolumn{9}{|c|}{ Local magnetic moments $\left(\mu_{\mathrm{B}}\right)$} & \multirow[b]{2}{*}{$T_{\mathrm{c}}(\mathrm{K})$} \\
\hline & $\mathrm{L}_{9}$ & $\mathrm{~L}_{8}$ & $\mathrm{~L}_{7}$ & $\mathrm{~L}_{6}$ & $\mathrm{~L}_{5}$ & $\mathrm{~L}_{4}$ & $\mathrm{~L}_{3}$ & $\mathrm{~L}_{2}$ & $\mathrm{~L}_{1}$ & \\
\hline $\mathrm{Fe}_{1} \mathrm{Co}_{1} \mathrm{Fe}_{1}$ & & & & & & & 2.70 & 0.0 & 2.41 & 482 \\
\hline $\mathrm{Fe}_{1} \mathrm{Co}_{2} \mathrm{Fe}_{1}$ & & & & & & 2.68 & 0.91 & 0.93 & 2.38 & 549 \\
\hline $\mathrm{Fe}_{1} \mathrm{Co}_{3} \mathrm{Fe}_{1}$ & & & & & 2.69 & 0.77 & 1.37 & 0.75 & 2.40 & 673 \\
\hline $\mathrm{Fe}_{1} \mathrm{Co}_{4} \mathrm{Fe}_{1}$ & & & & 2.69 & 0.80 & 1.31 & 1.29 & 0.80 & 2.39 & 783 \\
\hline $\mathrm{Fe}_{1} \mathrm{Co}_{5} \mathrm{Fe}_{1}$ & & & 2.69 & 0.80 & 1.32 & 1.22 & 1.31 & 0.80 & 2.39 & 755 \\
\hline $\mathrm{Fe}_{1} \mathrm{Co}_{6} \mathrm{Fe}_{1}$ & & 2.68 & 0.80 & 1.32 & 1.24 & 1.25 & 1.31 & 0.80 & 2.39 & 877 \\
\hline $\mathrm{Fe}_{1} \mathrm{Co}_{7} \mathrm{Fe}_{1}$ & 2.68 & 0.80 & 1.32 & 1.24 & 1.26 & 1.24 & 1.31 & 0.80 & 2.39 & 900 \\
\hline $\mathrm{Fe}_{2} \mathrm{Co}_{1} \mathrm{Fe}_{2}$ & & & & & 2.51 & 2.05 & 0.0 & 1.99 & 2.20 & 560 \\
\hline $\mathrm{Fe}_{2} \mathrm{Co}_{2} \mathrm{Fe}_{2}$ & & & & 2.51 & 2.01 & 0.96 & 0.94 & 1.96 & 2.21 & 598 \\
\hline $\mathrm{Fe}_{2} \mathrm{Co}_{3} \mathrm{Fe}_{2}$ & & & 2.51 & 2.02 & 0.88 & 1.33 & 0.87 & 1.97 & 2.21 & 649 \\
\hline $\mathrm{Fe}_{2} \mathrm{Co}_{4} \mathrm{Fe}_{2}$ & & 2.51 & 2.02 & 0.90 & 1.28 & 1.29 & 0.89 & 1.97 & 2.21 & 771 \\
\hline $\mathrm{Fe}_{2} \mathrm{Co}_{5} \mathrm{Fe}_{2}$ & 2.51 & 2.02 & 0.90 & 1.29 & 1.24 & 1.30 & 0.89 & 1.97 & 2.21 & 836 \\
\hline $\mathrm{Fe}_{3} \mathrm{Co}_{1} \mathrm{Fe}_{3}$ & & & 2.53 & 1.72 & 2.01 & 0.0 & 2.01 & 1.65 & 2.21 & 479 \\
\hline $\mathrm{Fe}_{3} \mathrm{Co}_{2} \mathrm{Fe}_{3}$ & & 2.53 & 1.73 & 1.98 & 0.94 & 0.94 & 1.98 & 1.66 & 2.21 & 485 \\
\hline $\mathrm{Fe}_{3} \mathrm{Co}_{3} \mathrm{Fe}_{3}$ & 2.53 & 1.73 & 1.99 & 0.85 & 1.33 & 0.86 & 2.00 & 1.66 & 2.21 & 607 \\
\hline $\mathrm{Co}_{1}$ & & & & & & & & & 1.66 & 1091 \\
\hline $\mathrm{Cu}_{7} \mathrm{Co}_{1}$ & & & & & & & & & 1.51 & 916 \\
\hline
\end{tabular}

a whole as it has a good $T_{\mathrm{c}}$. Interestingly a single Co layer embedded in a non-magnetic environment such as $\mathrm{Cu}$ does develop local moments with a magnetic moment of $1.51 \mu_{\mathrm{B}}$ and a $T_{\mathrm{c}}$ of $916 \mathrm{~K}$.

To understand the electronic origin of this phenomenon, in figure 1 we present the electronic densities of states (DOS) for some selected layers of the $\mathrm{Fe}_{1} \mathrm{Co}_{1} \mathrm{Fe}_{1} / \mathrm{Cu}(100)$ and $\mathrm{Fe}_{1} \mathrm{Co}_{3} \mathrm{Fe}_{1} / \mathrm{Cu}(100)$ films in the DLM state. We observe that, in the $\mathrm{Fe}_{1} \mathrm{Co}_{1} \mathrm{Fe}_{1} / \mathrm{Cu}(100)$ films, the exchange splitting of the bands vanishes for the Co layer, resulting in zero local magnetic moment. In the $\mathrm{Fe}_{1} \mathrm{Co}_{3} \mathrm{Fe}_{1} / \mathrm{Cu}(100)$ films, the central Co layer has a large exchange splitting very similar to that of $\mathrm{Fe}$ layers while for the two Co layers adjacent to the Fe layers the exchange splitting is less prominent, resulting in somewhat reduced local moments. This reduction in the exchange splitting in the Co layers close to the Fe layers is due to transfer of some of the electrons with spin anti-parallel to the local moment of the site from above the Fermi energy to just below the Fermi energy as is evident from figure 1(b). Also, the energy band of the electrons with spin parallel to the local moment of the site is somewhat broader on the Co layers close to the Fe layers.

There has been instances of single layers of ferromagnetic transition metals becoming magnetically dead in some nonmagnetic environments, such as $\mathrm{Co}$ in $\mathrm{Nb}$ and $\mathrm{Fe}$ in $\mathrm{Nb}$ [29-31], and $\mathrm{Ni}$ in $\mathrm{Pt}$ [32], etc, but none in a magnetic environment such as in the present case. However, our calculations of the direct correlation functions, i.e. the effective exchange interactions, $\mathcal{S}_{\mathrm{PQ}}^{(2)}\left(\mathbf{q}_{\|}=0\right)$, suggest that below $T_{\mathrm{c}}$ a ferromagnetic order becomes stable, although the local 
Table 2. Intralayer $\mathcal{S}_{\mathrm{PP}}^{(2)}\left(\mathbf{q}_{\|}=0\right)$ and interlayer

$\mathcal{S}_{\mathrm{PQ}}^{(2)}\left(\mathbf{q}_{\|}=0\right)(\mathrm{P} \neq \mathrm{Q})$ effective 'exchange interactions' in $\mathrm{meV}$ in the $\mathrm{Fe}_{1} \mathrm{Co}_{1} \mathrm{Fe}_{1} / \mathrm{Cu}(100)$ and $\mathrm{Fe}_{1} \mathrm{Co}_{3} \mathrm{Fe}_{1} / \mathrm{Cu}(100)$ films. Note that the layer $\mathrm{L}_{1}$ (the rightmost in the film title) is adjacent to the substrate and the leftmost layer is at the surface.

\begin{tabular}{|c|c|c|c|c|c|c|}
\hline \multirow[b]{2}{*}{ Films } & & \multicolumn{5}{|c|}{$\mathcal{S}_{\mathrm{PQ}}^{(2)}\left(\mathbf{q}_{\|}=0\right)(\mathrm{meV})$} \\
\hline & & $\mathrm{L}_{1}$ & $\mathrm{~L}_{2}$ & $\mathrm{~L}_{3}$ & $\mathrm{~L}_{4}$ & $\mathrm{~L}_{5}$ \\
\hline $\mathrm{Fe}_{1} \mathrm{Co}_{1} \mathrm{Fe}_{1}$ & $\begin{array}{l}\mathrm{L}_{1} \\
\mathrm{~L}_{2} \\
\mathrm{~L}_{3}\end{array}$ & $\begin{array}{r}115.2 \\
0.0 \\
16.0\end{array}$ & $\begin{array}{l}0.0 \\
0.0 \\
0.0\end{array}$ & $\begin{array}{r}16.0 \\
0.0 \\
97.0\end{array}$ & & \\
\hline $\mathrm{Fe}_{2} \mathrm{Co}_{1} \mathrm{Fe}_{2}$ & $\begin{array}{l}\mathrm{L}_{1} \\
\mathrm{~L}_{2} \\
\mathrm{~L}_{3} \\
\mathrm{~L}_{4} \\
\mathrm{~L}_{5}\end{array}$ & $\begin{array}{r}116.1 \\
-0.5 \\
0.0 \\
2.6 \\
2.1\end{array}$ & $\begin{array}{r}-0.5 \\
17.8 \\
0.0 \\
-16.1 \\
2.0\end{array}$ & $\begin{array}{l}0.0 \\
0.0 \\
0.0 \\
0.0 \\
0.0\end{array}$ & $\begin{array}{r}2.6 \\
-16.1 \\
0.0 \\
39.6 \\
60.2\end{array}$ & $\begin{array}{r}2.1 \\
2.0 \\
0.0 \\
60.2 \\
109.5\end{array}$ \\
\hline $\mathrm{Fe}_{1} \mathrm{Co}_{2} \mathrm{Fe}_{1}$ & $\begin{array}{l}\mathrm{L}_{1} \\
\mathrm{~L}_{2} \\
\mathrm{~L}_{3} \\
\mathrm{~L}_{4}\end{array}$ & $\begin{array}{r}112.9 \\
49.0 \\
-13.9 \\
10.9\end{array}$ & $\begin{array}{r}49.0 \\
43.2 \\
62.6 \\
-11.0\end{array}$ & $\begin{array}{r}-13.9 \\
62.6 \\
30.0 \\
76.2\end{array}$ & $\begin{array}{r}10.9 \\
-11.0 \\
76.2 \\
-530.4\end{array}$ & \\
\hline $\mathrm{Fe}_{1} \mathrm{Co}_{3} \mathrm{Fe}_{1}$ & $\begin{array}{l}\mathrm{L}_{1} \\
\mathrm{~L}_{2} \\
\mathrm{~L}_{3} \\
\mathrm{~L}_{4} \\
\mathrm{~L}_{5}\end{array}$ & $\begin{array}{r}111.4 \\
46.1 \\
-8.4 \\
1.6 \\
0.0\end{array}$ & $\begin{array}{r}46.1 \\
24.5 \\
63.2 \\
-3.9 \\
2.1\end{array}$ & $\begin{array}{r}-8.4 \\
63.2 \\
98.0 \\
68.9 \\
-5.5\end{array}$ & $\begin{array}{r}1.6 \\
-3.9 \\
68.9 \\
19.4 \\
69.4\end{array}$ & $\begin{array}{r}0.0 \\
2.1 \\
-5.5 \\
69.4 \\
103.9\end{array}$ \\
\hline
\end{tabular}

moment of the Co atoms in some films turns out to be zero in the DLM state. We present the values of the interlayer $(\mathrm{P} \neq \mathrm{Q})$ as well as the intralayer $(\mathrm{P}=\mathrm{Q})$ effective exchange interactions, $\mathcal{S}_{\mathrm{PQ}}^{(2)}\left(\mathbf{q}_{\|}=0\right)$, for the $\mathrm{Fe}_{1} \mathrm{Co}_{1} \mathrm{Fe}_{1} / \mathrm{Cu}(100)$, $\mathrm{Fe}_{2} \mathrm{Co}_{1} \mathrm{Fe}_{2} / \mathrm{Cu}(100), \quad \mathrm{Fe}_{1} \mathrm{Co}_{2} \mathrm{Fe}_{1} / \mathrm{Cu}(100)$ and $\mathrm{Fe}_{1} \mathrm{Co}_{3} \mathrm{Fe}_{1} /$ $\mathrm{Cu}(100)$ films in table 2. We observe that, for films with only one Co layer, both the interlayer as well as the intralayer exchange interactions for the Co layer are zero, indicating that both the Co-Co as well as the Co-Fe couplings are negligible. This is in accordance with the vanishing of the local moment of the Co atoms in the $\mathrm{Fe}_{1} \mathrm{Co}_{1} \mathrm{Fe}_{1} / \mathrm{Cu}(100)$ film. We also note that in the $\mathrm{Fe}_{n} \mathrm{Co}_{1} \mathrm{Fe}_{n} / \mathrm{Cu}(100)$ films for $n>1$, the intralayer $\mathrm{Fe}-\mathrm{Fe}$ coupling is strongly ferromagnetic and the Fe layers near the surface are coupled ferromagnetically, whereas the Fe layers on either side of the Co layer are coupled antiferromagnetically. However, the nature of the magnetic interactions are quite different in the $\mathrm{Fe}_{1} \mathrm{Co}_{n} \mathrm{Fe}_{1} / \mathrm{Cu}$ (100) films for $n>1$, and more importantly, the magnetic couplings change dramatically between $n=2$ and 3 . For the $\mathrm{Fe}_{1} \mathrm{Co}_{2} \mathrm{Fe}_{1} / \mathrm{Cu}(100)$ film the $\mathrm{Fe}$ layer at the surface shows a strong tendency to antiferromagnetic ordering, while all the other dominant interactions are ferromagnetic. This is in contrast with the $\mathrm{Fe}_{1} \mathrm{Co}_{3} \mathrm{Fe}_{1} / \mathrm{Cu}$ (100) film in which all the dominant magnetic interactions, including the one for the surface layer, are ferromagnetic. This trend, together with the quite weak interlayer magnetic interactions beyond the firstneighbour layers, prevails for thicker films as well.

To get a more detailed insight of the unusual magnetism of the $\mathrm{Fe}_{1} \mathrm{Co}_{1} \mathrm{Fe}_{1} / \mathrm{Cu}(100)$ film, we performed further calculations for several ordered (collinear) magnetic configurations of this system. In line with the predictions of the DLM theory, the overall ferromagnetic state has the lowest total energy. However, in this state the Co atoms have a magnetic moment of

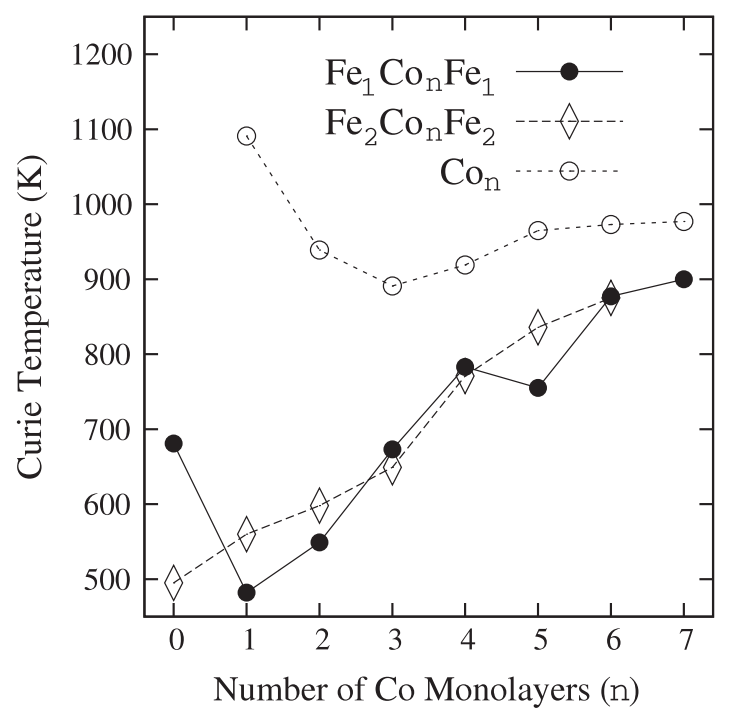

Figure 2. Curie temperature $T_{\mathrm{c}}$ of $\mathrm{Fe}_{1} \mathrm{Co}_{1} \mathrm{Fe}_{1} / \mathrm{Cu}(100)$ (filled circles), $\mathrm{Fe}_{2} \mathrm{Co}_{n} \mathrm{Fe}_{2} / \mathrm{Cu}(100)$ (diamonds) and $\mathrm{Co}_{n} / \mathrm{Cu}$ (100) (open circles) films for $n=0,1,2,3,4,5,6$ and 7 . The lines are a guide to the eye.

$1.54 \mu_{\mathrm{B}}$. We also find that, in the metastable magnetic configuration, in which one of the Fe layers has an in-plane antiferromagnetic order and the other one is ferromagnetic, the local moment of Co atoms decreases to $1.15 \mu_{\mathrm{B}}$ or $1.03 \mu_{\mathrm{B}}$, depending on whether the ferromagnetic Fe layer is at the surface or at the interface. Furthermore, if both the Fe layers are forced to have in-plane antiferromagnetic order then the local moment on the Co layer vanishes altogether. These observations imply that the local spin polarization of the Co atoms is very sensitive to the magnetic order in the adjacent Fe layers. In such a case it is difficult to provide a consistent description of the ground state from the paramagnetic state described within the DLM scheme, as it is based on the premise that longitudinal spin fluctuations are of minor importance.

In figure 2 we show the Curie temperatures of $\mathrm{Co}_{n} / \mathrm{Cu}(100), \mathrm{Fe}_{1} \mathrm{Co}_{n} \mathrm{Fe}_{1} / \mathrm{Cu}(100)$ and $\mathrm{Fe}_{2} \mathrm{Co}_{n} \mathrm{Fe}_{2} / \mathrm{Cu}(100)$ films as a function of Co layer thickness $(n)$. In the $\mathrm{Fe}_{1} \mathrm{Co}_{n} \mathrm{Fe}_{1} / \mathrm{Cu}(100)$ film, the $T_{\mathrm{c}}$ drops by almost $200 \mathrm{~K}$ when the first Co layer is placed between the two Fe layers and this can be attributed to the vanishing of the magnetic interactions between the $\mathrm{Fe}$ and $\mathrm{Co}$ layers as well as that between the Co atoms in the single Co layer. Thereafter addition of each Co layer between the Fe layers increases the $T_{\mathrm{c}}$ except for a slight drop for $n=5$. This almost monotonically increasing behaviour is probably due to the strengthening of the intralayer as well as interlayer ferromagnetic couplings. Remarkably, however, in the $\mathrm{Fe}_{2} \mathrm{Co}_{n} \mathrm{Fe}_{2} / \mathrm{Cu}(100)$ films the $T_{\mathrm{c}}$ for $n=1$ is higher than that for $n=0$, although the Co layer for $n=1$ has a zero local moment. This implies that the $T_{\mathrm{c}}$ 's are more closely related to the magnetic interactions between the atoms rather than to their local moments. We also note that the bare $\mathrm{Co}_{n} / \mathrm{Cu}(100)$ films have higher $T_{\mathrm{c}}$ 's than the corresponding sandwich structure $\mathrm{Fe}_{m} \mathrm{Co}_{n} \mathrm{Fe}_{m} / \mathrm{Cu}(100)$. 
Table 3. The local magnetic moments on different layers and the Curie temperatures of $\mathrm{Co}_{m} \mathrm{Fe}_{n} \mathrm{Co}_{m} / \mathrm{Cu}(100)$ films. Note that the layer $\mathrm{L}_{1}$ (the rightmost in the film title) is adjacent to the substrate and the leftmost layer is at the surface.

\begin{tabular}{|c|c|c|c|c|c|c|c|c|c|c|}
\hline \multirow[b]{2}{*}{ Films } & \multicolumn{9}{|c|}{ Local magnetic moments $\left(\mu_{\mathrm{B}}\right)$} & \multirow[b]{2}{*}{$T_{\mathrm{c}}(\mathrm{K})$} \\
\hline & $\mathrm{L}_{9}$ & $\mathrm{~L}_{8}$ & $\mathrm{~L}_{7}$ & $\mathrm{~L}_{6}$ & $\mathrm{~L}_{5}$ & $\mathrm{~L}_{4}$ & $\mathrm{~L}_{3}$ & $\mathrm{~L}_{2}$ & $\mathrm{~L}_{1}$ & \\
\hline $\mathrm{Co}_{1} \mathrm{Fe}_{1} \mathrm{Co}_{1}$ & & & & & & & 1.41 & 2.19 & 1.13 & 485 \\
\hline $\mathrm{Co}_{1} \mathrm{Fe}_{2} \mathrm{Co}_{1}$ & & & & & & 1.44 & 2.03 & 1.94 & 1.16 & 654 \\
\hline $\mathrm{Co}_{1} \mathrm{Fe}_{3} \mathrm{Co}_{1}$ & & & & & 1.45 & 2.02 & 1.71 & 1.94 & 1.16 & 629 \\
\hline $\mathrm{Co}_{1} \mathrm{Fe}_{4} \mathrm{Co}_{1}$ & & & & 1.44 & 2.02 & 1.71 & 1.71 & 1.94 & 1.16 & 584 \\
\hline $\mathrm{Co}_{1} \mathrm{Fe}_{5} \mathrm{Co}_{1}$ & & & 1.44 & 2.02 & 1.71 & 1.71 & 1.71 & 1.93 & 1.16 & 625 \\
\hline $\mathrm{Co}_{1} \mathrm{Fe}_{6} \mathrm{Co}_{1}$ & & 1.44 & 2.02 & 1.71 & 1.71 & 1.71 & 1.71 & 1.93 & 1.16 & 584 \\
\hline $\mathrm{Co}_{1} \mathrm{Fe}_{7} \mathrm{Co}_{1}$ & 1.44 & 2.02 & 1.71 & 1.71 & 1.72 & 1.72 & 1.71 & 1.94 & 1.16 & 587 \\
\hline $\mathrm{Co}_{2} \mathrm{Fe}_{1} \mathrm{Co}_{2}$ & & & & & 1.67 & 0.85 & 2.25 & 0.79 & 1.43 & 807 \\
\hline $\mathrm{Co}_{2} \mathrm{Fe}_{2} \mathrm{Co}_{2}$ & & & & 1.66 & 0.91 & 1.99 & 2.00 & 0.85 & 1.43 & 866 \\
\hline $\mathrm{Co}_{2} \mathrm{Fe}_{3} \mathrm{Co}_{2}$ & & & 1.66 & 0.90 & 1.99 & 1.69 & 2.00 & 0.83 & 1.43 & 804 \\
\hline $\mathrm{Co}_{2} \mathrm{Fe}_{4} \mathrm{Co}_{2}$ & & 1.66 & 0.90 & 1.99 & 1.70 & 1.70 & 2.00 & 0.83 & 1.43 & 803 \\
\hline $\mathrm{Co}_{2} \mathrm{Fe}_{5} \mathrm{Co}_{2}$ & 1.66 & 0.90 & 1.99 & 1.70 & 1.71 & 1.70 & 2.00 & 0.83 & 1.43 & 803 \\
\hline $\mathrm{Co}_{3} \mathrm{Fe}_{1} \mathrm{Co}_{3}$ & & & 1.62 & 1.30 & 0.85 & 2.24 & 0.86 & 1.25 & 1.38 & 906 \\
\hline $\mathrm{Co}_{3} \mathrm{Fe}_{2} \mathrm{Co}_{3}$ & & 1.62 & 1.29 & 0.88 & 2.00 & 2.00 & 0.89 & 1.24 & 1.38 & 905 \\
\hline $\mathrm{Co}_{3} \mathrm{Fe}_{3} \mathrm{Co}_{3}$ & 1.62 & 1.29 & 0.87 & 2.00 & 1.70 & 2.00 & 0.87 & 1.24 & 1.38 & 904 \\
\hline & & & & & & & & & 2.67 & 1224 \\
\hline $\mathrm{Cu}_{7} \mathrm{Fe}_{1}$ & & & & & & & & & 2.46 & 1142 \\
\hline
\end{tabular}

\section{2. $\mathrm{Co}_{m} \mathrm{Fe}_{n} \mathrm{Co}_{m} / \mathrm{Cu}(100)$ films}

These multilayers are in the same class of fundamental importance as $\mathrm{Fe}_{m} \mathrm{Co}_{n} \mathrm{Fe}_{m} / \mathrm{Cu}(100)$ films. These are also clean films because of the complete immiscibility of $\mathrm{Co}$ and $\mathrm{Cu}$ in the bulk phase and a rather small lattice mismatch $[35,36]$. Our previous work on $\mathrm{Co}_{n} \mathrm{Fe}_{m} / \mathrm{Cu}(100)$ films showed that the thickness of the Co layer on Fe films helps in stabilizing the ferromagnetic behaviour of the films resulting in an increase of the local magnetic moments as well as the Curie temperature of the films. In the case of the $\mathrm{Fe}_{m} \mathrm{Co}_{n} \mathrm{Fe}_{m} / \mathrm{Cu}(100)$ films the local magnetic moment on Co layers is diminished. In this subsection we present the results of $\mathrm{Co}_{m} \mathrm{Fe}_{n} \mathrm{Co}_{m} / \mathrm{Cu}$ (100) films with $n$ and $m$ varying between 0 and 3 . The local magnetic moments and the Curie temperatures of various films are presented in table 3. As far as the local magnetic moments are concerned, there is a striking contrast between this system and the $\mathrm{Fe}_{m} \mathrm{Co}_{n} \mathrm{Fe}_{m} / \mathrm{Cu}(100)$ system. In the present case, the local magnetic moments on Fe layers adjacent to the Co layers are larger compared to those on Fe layers which are further from the Co layers. This is because of the charge transfer between the nearest-neighbour $\mathrm{Fe}$ and $\mathrm{Co}$ layers. We observe that the Co layer loses some electrons (about 0.25 electrons/atom) with their spins parallel to the local magnetic moment to the Fe layer while it gains some electrons (about 0.25 electrons/atom) with their spins anti-parallel to the local magnetic moment from the Fe layer, thereby reducing the local moment of $\mathrm{Co}$ atoms and enhancing that of the $\mathrm{Fe}$ atoms. This same mechanism is at work in the $\mathrm{Fe}_{m} \mathrm{Co}_{n} \mathrm{Fe}_{m} / \mathrm{Cu}(100)$ films as well. Therefore the local magnetic moments in a single Fe layer sandwiched between two Co layers is much enhanced whereas those in a single Co layer between two Fe layers vanishes. We present the values of the interlayer $(\mathrm{P} \neq \mathrm{Q})$ as well as the intralayer $(\mathrm{P}=\mathrm{Q})$ effective exchange interactions, $\mathcal{S}_{\mathrm{PQ}}^{(2)}\left(\mathbf{q}_{\|}=0\right)$, for the $\mathrm{Co}_{1} \mathrm{Fe}_{1} \mathrm{Co}_{1} / \mathrm{Cu}(100)$ and $\mathrm{Co}_{1} \mathrm{Fe}_{3} \mathrm{Co}_{1} / \mathrm{Cu}(100)$ films in table 4. Here again we find that the magnetic interactions are confined mostly to nearest
Table 4. Intralayer $\mathcal{S}_{\mathrm{PP}}^{(2)}\left(\mathbf{q}_{\|}=0\right)$ and interlayer $\mathcal{S}_{\mathrm{PQ}}^{(2)}\left(\mathbf{q}_{\|}=0\right)(\mathrm{P} \neq \mathrm{Q})$ effective 'exchange interactions' in $\mathrm{meV}$ in the $\mathrm{Co}_{1} \mathrm{Fe}_{1} \mathrm{Co}_{1} / \mathrm{Cu}(100)$ and $\mathrm{Co}_{1} \mathrm{Fe}_{3} \mathrm{Co}_{1} / \mathrm{Cu}(100)$ films. Note that the layer $\mathrm{L}_{1}$ (the rightmost in the film title) is adjacent to the substrate and the leftmost layer is at the surface.

\begin{tabular}{|c|c|c|c|c|c|c|}
\hline \multirow[b]{2}{*}{ Films } & & \multicolumn{5}{|c|}{$\mathcal{S}_{\mathrm{PQ}}^{(2)}\left(\mathbf{q}_{\|}=0\right)(\mathrm{meV})$} \\
\hline & & $\mathrm{L}_{1}$ & $\mathrm{~L}_{2}$ & $\mathrm{~L}_{3}$ & $\mathrm{~L}_{4}$ & $\mathrm{~L}_{5}$ \\
\hline $\mathrm{Co}_{1} \mathrm{Fe}_{1} \mathrm{Co}_{1}$ & $\begin{array}{l}\mathrm{L}_{1} \\
\mathrm{~L}_{2} \\
\mathrm{~L}_{3}\end{array}$ & $\begin{array}{r}76.6 \\
37.3 \\
-8.8\end{array}$ & $\begin{array}{l}37.3 \\
13.6 \\
49.6\end{array}$ & $\begin{array}{r}-8.8 \\
49.6 \\
101.9\end{array}$ & & \\
\hline $\mathrm{Co}_{1} \mathrm{Fe}_{3} \mathrm{Co}_{1}$ & $\begin{array}{l}\mathrm{L}_{1} \\
\mathrm{~L}_{2} \\
\mathrm{~L}_{3} \\
\mathrm{~L}_{4} \\
\mathrm{~L}_{5}\end{array}$ & $\begin{array}{r}80.4 \\
41.9 \\
-0.9 \\
0.3 \\
1.2\end{array}$ & $\begin{array}{r}41.9 \\
15.4 \\
-2.0 \\
6.6 \\
0.3\end{array}$ & $\begin{array}{r}-0.9 \\
-2.0 \\
21.8 \\
-2.6 \\
2.7\end{array}$ & $\begin{array}{r}0.3 \\
6.6 \\
-2.6 \\
29.8 \\
81.3\end{array}$ & $\begin{array}{r}1.2 \\
0.3 \\
2.7 \\
81.3 \\
112.5\end{array}$ \\
\hline
\end{tabular}

neighbours and are ferromagnetic in nature. It should be noted that the interlayer Fe-Fe couplings are very weak even for nearest-neighbour layers. Therefore, introducing more Fe layers does not enhance the overall ferromagnetic nature of the films. So it is expected that the $T_{\mathrm{c}}$ will be more or less stable when more Fe layers are added between the two Co layers.

The Curie temperatures of the films also show an interesting behaviour. In figure 3 we show the Curie temperatures of $\mathrm{Fe}_{n} / \mathrm{Cu}(100), \mathrm{Co}_{1} \mathrm{Fe}_{n} \mathrm{Co}_{1} / \mathrm{Cu}(100)$ and $\mathrm{Co}_{2} \mathrm{Fe}_{n} \mathrm{Co}_{2} / \mathrm{Cu}(100)$ films as a function of $\mathrm{Fe}$ layer thickness $(n)$. For a pure $\mathrm{Fe}$ film the $T_{\mathrm{c}}$ drops from a high $1200 \mathrm{~K}$ for a single Fe layer to $680 \mathrm{~K}$ for two Fe layers and after that it stabilizes around $480 \mathrm{~K}$ for thicker films. In contrast to the $\mathrm{Fe}_{m} \mathrm{Co}_{n} \mathrm{Fe}_{m} / \mathrm{Cu}(100)$ films the $\mathrm{Co}_{m} \mathrm{Fe}_{n} \mathrm{Co}_{m} / \mathrm{Cu}(100)$ films, except for the single $\mathrm{Fe}$ layer, tend to have a higher $T_{\mathrm{c}}$ than the pure Fe films for all thicknesses. Also, for the sandwiched Fe films, the $T_{\mathrm{c}}$ exhibits an interesting oscillatory behaviour as the thickness of the Fe layer between the two Co layers is increased, in contrast to the $\mathrm{Fe}_{m} \mathrm{Co}_{n} \mathrm{Fe}_{m} / \mathrm{Cu}(100)$ films. The $T_{\mathrm{c}}$ has a large value when there are no Fe layers between the Co layers, it decreases 


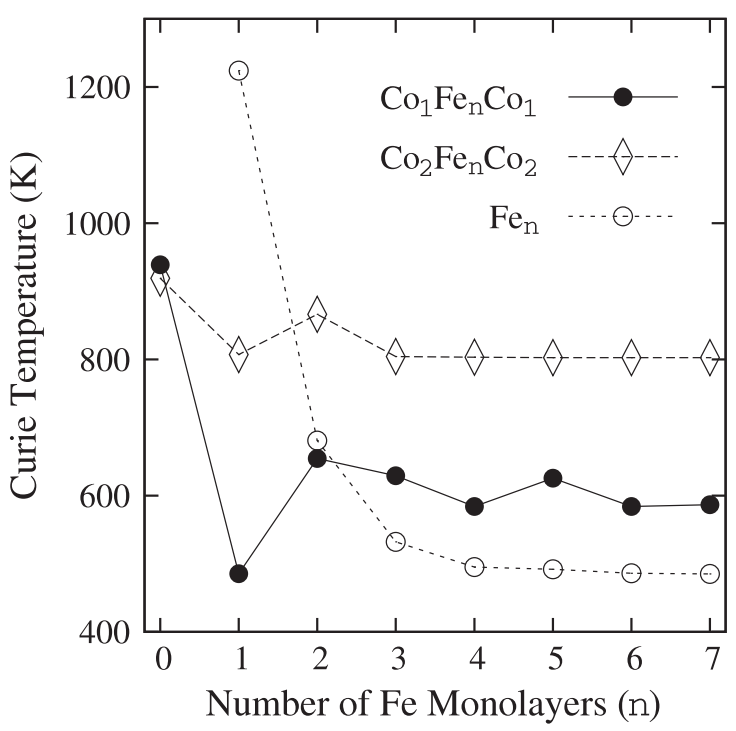

Figure 3. Curie temperature $T_{\mathrm{c}}$ of $\mathrm{Co}_{1} \mathrm{Fe}_{n} \mathrm{Co}_{1} / \mathrm{Cu}$ (100) (filled circles), $\mathrm{Co}_{2} \mathrm{Fe}_{n} \mathrm{Co}_{2} / \mathrm{Cu}(100)$ (diamonds) and $\mathrm{Fe}_{n} / \mathrm{Cu}(100)$ (open circles) films for $n=0,1,2,3,4,5,6$ and 7 . The lines are a guide to the eye.

sharply when a single Fe layer is inserted between the Co layers and thereafter it oscillates as the Fe layer thickness is increased and finally apparently stabilizes when the Fe layer thickness becomes more than six. Our results therefore show that an $\mathrm{Fe}$ film sandwiched between Co layers has a higher $T_{\mathrm{c}}$ than that of the corresponding bare Fe film whereas a Co film sandwiched between Fe layers has a lower $T_{\mathrm{c}}$ than that of the corresponding bare Co film. It would be interesting to see whether these findings can be tested by experimental measurements.

\section{Conclusions}

We have calculated the local magnetic moments and investigated the onset of ferromagnetic order in some $\mathrm{Co}_{m} \mathrm{Fe}_{n} \mathrm{Co}_{m} / \mathrm{Cu}(100)$ and $\mathrm{Fe}_{m} \mathrm{Co}_{n} \mathrm{Fe}_{m} / \mathrm{Cu}(100)$ films from a first-principles electronic structure point of view. The local moment of a Co monolayer placed between two Fe layers becomes zero in the paramagnetic state while those on the $\mathrm{Fe}$ layers are enhanced. This is due to charge transfer between the $\mathrm{Fe}$ and Co layers. The Curie temperature and the magnetic interactions as well as the variation of the paramagnetic spin susceptibility of the films strongly depend upon the structure and thickness of the films and show some interesting behaviour as a function of the structure of the film.

\section{Acknowledgments}

This work was supported by Kuwait University, Research Administration, through Research Grant no. SP 02/02 and the Hungarian National Scientific Research Foundation through contract nos. K68312 and K77771.

\section{References}

[1] Bader S D 2006 Rev. Mod. Phys. 781

[2] Himpsel F J, Ortega J E, Mankey G J and Willis R F 1998 Advances in Physics: Magnetic Nanostructures vol 47, ed D Sherrington (London: Taylor and Francis) p 511
[3] Krusin-Elbaum L, Shinauchi T, Argyle B, Gignac L and Weller D 2001 Nature 410444

[4] Li S P, Lew W S, Bland J A C, Lopez-Diaz L, Natali M, Vaz C A F and Chen Y 2002 Nature 415600

[5] Weller D and Moser A 1999 IEEE Trans. Magn. 354423

[6] Parkin S S P, Jiang X, Kaiser C, Panchula A, Roche K and Samant M 2003 Proc. IEEE 91661

[7] Zhao H G, Won C, Wu Y Z, Scholl A, Doran A and Qiu Z Q 2004 Phys. Rev. B 70024423

[8] Hubbard J 1979 Phys. Rev. B 204584

[9] Hasegawa H 1979 J. Phys. Soc. Japan 461504

[10] Gyorffy B L, Pindor A J, Staunton J B, Stocks G M and Winter H 1985 J. Phys. F: Met. Phys. 151337

[11] Staunton J B, Gyorffy B L, Stocks G M and Wadsworth J 1985 J. Phys. F: Met. Phys. 151387

[12] Staunton J B, Gyorffy B L, Stocks G M and Wadsworth J 1986 J. Phys. F: Met. Phys. 161761

[13] Staunton J B and Gyorffy B L 1992 Phys. Rev. Lett. 69371

[14] Razee S S A, Staunton J B, Szunyogh L and Gyorffy B L 2002 Phys. Rev. Lett. 88147201

[15] Razee S S A, Staunton J B, Szunyogh L and Gyorffy B L 2002 Phys. Rev. B 66094415

[16] Razee S S A, Staunton J B, Szunyogh L and Gyorffy B L 2002 Physica B 318316

[17] Razee S S A 2004 J. Magn. Magn. Mater. 274-276 804

[18] Vollmer R, van Dijken S, Schleberger M and Kirschner J 2000 Phys. Rev. B 611303

[19] Staunton J B, Ostanin S, Razee S S A, Gyorffy B L, Szunyogh L, Ginatempo B and Bruno E 2004 Phys. Rev. Lett. 93257204

[20] Staunton J B, Szunyogh L, Buruzs A, Gyorffy B L, Ostanin S and Udvardi L 2006 Phys. Rev. B 74144411

[21] Buruzs A, Weinberger P, Szunyogh L, Udvardi L, Chleboun P I, Fischer A M and Staunton J B 2007 Phys. Rev. B 76064417

[22] Buruzs A, Szunyogh L, Udvardi L, Weinberger P and Staunton J B 2007 J. Magn. Magn. Mater. 316 E371

[23] Hughes I D, Däne M, Ernst A, Hergert W, Lüders M, Poulter J, Staunton J B, Svane A, Szotek Z and Temmerman W M 2007 Nature $\mathbf{4 4 6} 650$

[24] Hughes I D, Däne M, Ernst A, Hergert W, Lüders M, Staunton J B, Szotek Z and Temmerman W M 2008 New J. Phys. 10063010

[25] Szunyogh L, Ujfalussy B and Weinberger P 1995 Phys. Rev. B $\mathbf{5 1} 9552$

[26] Weinberger P and Szunyogh L 2000 Comput. Mater. Sci. 17414

[27] Zabloudil J, Hammerling R, Szunyogh L and Weinberger P 2004 Electron Scattering in Solid Matter: a Theoretical and Computational Treatise (Berlin: Springer)

[28] Burkert T, Nordstrom L, Eriksson O and Heinonen O 2004 Phys. Rev. Lett. 93027203

[29] Mühge T, Theis-Brohl K, Zabel H, Garifyanov N N, Goryunov Y V, Garifullin I A and Kahliullin G G 1997 Phys. Rev. B 558945

[30] Mühge T, Theis-Brohl K, Westerholt K, Garifyanov N N, Goryunov Y V, Garifullin I A and Kahliullin G G 1998 Phys. Rev. B 575071

[31] Jamet M, Dupuis V, Mélinon P, Guiraud G, Pérez A, Wernsdorfer W, Traverse A and Baguenard B 2000 Phys. Rev. B 62493

[32] Kim S-K, Jeong J-R, Kortright J B and Shin S-C 2001 Phys. Rev. B 64052406

[33] Jensen P and Bennemann K H 2006 Surf. Sci. 61129

[34] Capelle K 2006 Braz. J. Phys. 361318

[35] Germer R, Dürr W, Krewer W, Pescia D and Gudat W 1988 Appl. Phys. A 47393

[36] Dittschar A, Kuch W, Zharnikov M and Schneider C M 2000 J. Magn. Magn. Mater. 212307 